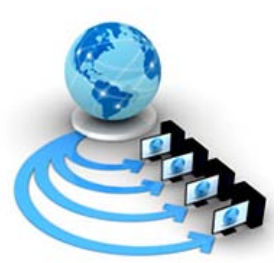

Volume 8, No. 8, September-October 2017

International Journal of Advanced Research in Computer Science

RESEARCH PAPER

\title{
A SURVEY: STUDY ABOUT BIOMETRIC IRIS DETECTION TECHNIQUE IN DIGITAL IMAGE PROCESSING
}

\author{
Richa Bajpai \\ M. Tech (Cyber Security) \\ Madhav Institute of Technology \&Science, \\ Gwalior, M.P., India
}

\author{
Prof. Punit Kumar Johari \\ Asst. Prof., Dept of CSE \& IT, \\ Madhav Institute of Technology \& Science, \\ Gwalior, M.P., India
}

\begin{abstract}
A safe and secure space is significant for everyone in their life. Biometric approaches are adapted for the proof of identity of individuals are currently increasingly being used in routine tasks like security areas, medical checkups etc. Here a study on iris recognition system for biometric identification due to attraction of having almost zero probability of finding similar iris to another even in genetic identical twins. As it is an external organ and it remains same throughout the life, allures towards other means of biometric identity systems. In this paper, brief detailing about work done previously in field of iris recognition. As proceeding with iris recognition, steps of image acquisition, preprocessing of captured image, segmentation, feature extraction, matching need to be performed.
\end{abstract}

Keywords: Iris recognition, segmentation, security, Biometric, Hough transform, counter measurement, IRIS, etc.

\section{INTRODUCTION:}

Enhancing the infrastructure against security threats will be attain by biometric authentication. Individual's identity measurement is classified into two major fields: physiological characteristics and behavioral characteristics [8]. Face, fingerprint, palm, retina, iris etc are come under the physiological classification whereas signature, keystroke based identification come under behavioral means of identification. Sometimes alliance of both can be used as using voice recognition. Biometric based iris recognition technology has significance after cogitation over the other biometric technologies. Iris recognition is used for verification as well as identification of an individual accurately. Iris has the advantages of uniqueness, stability, high recognition rate, non infringing etc[12]. Iris is the annular ring between sclera and pupil[8]. Individual's iris patterns unique and mechanically distinct although its coloration and structure. The French ophthalmologist Alphonse Bertillon [9] seems to be the first to propose the use of iris pattern (color) as a basis for personal identification. Iris recognition can be performed in following ways: texture based, appearance based, feature extraction based[10] and many more other methods. It takes very less time slice for recognize the individual. It consumes about approximately $471 \mathrm{~ms}$ [7] for recognition. Performance is to be evaluated over EER[8], FAR[8], FRR[8], FTE, Overall Accuracy (OA)[14] etc. different versions of CASIA[8], UBIRIS[11] databases are practiced for iris recognition. Through exercising with iris recognition, high rate of accuracy and confidentiality will accomplished.

\section{IRIS RECOGNITION TECHNOLOGY:}

Iris recognition progresses[14] under the following:

- Image Acquisition
- $\quad$ Preprocessing

- Feature Extraction

- Template Matching

- Result

Image Acquisition:

A subject merely needs to stand still and face forward with their head. Standing should be without head tilt [13] in idol situation. A distance of approximately 0.38 to $0.76 \mathrm{~m}$ between image capturing systems, all measured from the front-center of the acquisition rig.

\section{Preprocessing:}

Unwanted portions[8] are also captured during acquisition which confronts dubiousness in computation for recognition. So there is necessity for preprocessing before feature extraction due to inclusion of eyelashes, eyelids[8] etc. Eyelids, eyelashes are considered as noise is needed to exclude for recognition correctly. Localization and normalization are executed to accomplish noise free image. Isolation of iris region from rest of portion of eye is accomplished through localization of iris in captured image. Extracting the iris regions having same constant dimensions Produced by normalization due to reason of capturing the same eye image under different conditions. Characteristic features will be accumulated during image normalizing at the same spatial location. Distortion of an image is reduced by going with normalization of an image. Iris segmentation accumulates region of interest ROI.

\section{Feature Extraction:}

Now we need to examine the features such as textures of the iris for distinguishing features within these zones. Most prominent features are $\mathrm{x}, \mathrm{y}$ coordinates, radius, shape, size intensity value[15] of iris for recognize the iris. Detection of the geometrical curves like dots, lines, 
circle, ellipses etc. achieved by means of feature extraction. Statistical features [8] extraction is used in assessing the correlation between the adjacent pixels.

\section{Template Matching:}

Iris matching can be understood as a three-stage process as follows.

1. The first stage is concerned with establishing a spatial correspondence between two iris signatures that are to be compared.

2. Given correspondence, the second stage is concerned with quantifying the goodness of match between two iris signatures.

3. The third stage is concerned with making a decision about whether or not two signatures derive from the same physical iris, based on the goodness of match [5].

\section{Matching:}

Final step in iris recognition process takes place as matching with stored template in database in either identification or verification or both. The authentic individual will be allowed and refused imposter one on erroneous entry by the identification system [8]:

$D_{i}^{*}=\min D\left(F c, F^{i} c\right), i=1, N$

Where,

$\mathrm{Fc}=$ Feature of the input pattern $\mathrm{Fci}=$ Feature of the ith pattern from the database $\mathrm{N}=$ Number of patterns in the database.

In such a process, there is a most similar pattern which is assigned as $i^{*}$, to the database pattern. $i^{*}$ is the pattern which has a minimum distance value of $\mathrm{Di}^{*}$.

\section{AN IRIS RECOGNITION SYSTEM:}

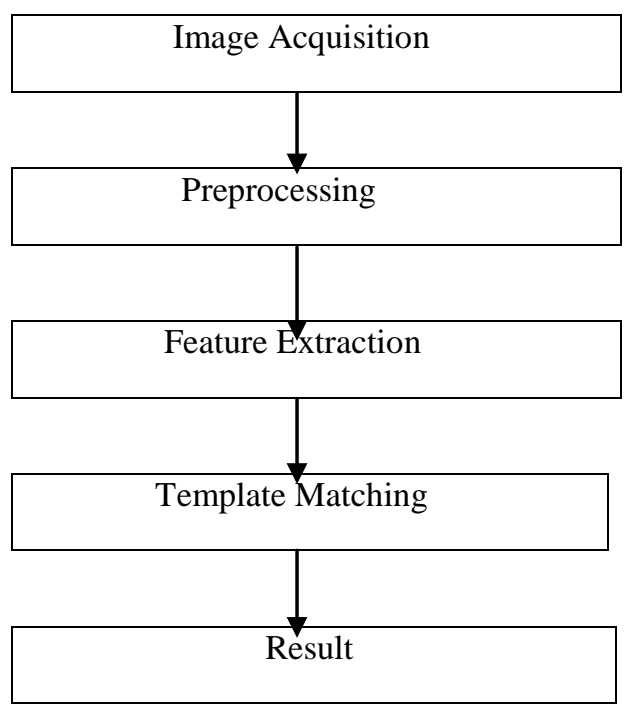

Fig.: Iris Recognition System

\section{LITERATURE SURVEY}

Recent perceptible readings in personal identification based on the patterns and colors of the iris, below some at these researches:
Srinivasa Kumar Devireddy, G.Ramaswamy, proposed a novel approach for accurate human recognition identification through iris recognition using bit plane slicing [1] and normalization. Iris boundaries are recognized by using simple methods and the less complex and faster algorithms than previous algorithms and it eliminates pupilary noises and reflections. Homogenization [1] removes specularities of the pupil. By solving these parameters in circle equation, they can recognize limbic boundary (outer boundary) accurately. The region between inner and outer boundary is iris, it is in the polar form [1] and converted into linear form by converting the polar coordinate system to Cartesian coordinate system, then converting the iris region from Cartesian coordinates to the normalized non concentric polar representation we get normalized image. [1]

Matsoso Samuel Monaheng1, Padmaja Kuruba use horizontal and vertical derivatives for edge mapping results 95.6\% whereas pervious results $88.1 \%$. iris recognition comes under physiological classification of biometric identification system. Digital image is used for identification. It has advantages such as simplicity, accuracy, high speed of computation because of sample size. On the unique pattern of iris in identification and verification of an individual, iris recognition works under segmentation, normalisation, feature extraction and matching phases. Separation iris region from entire captured image is done through segmentation, normalisation for fixing the dimension for comparison, and feature extraction draws bio templates and matches with reference template. Iris recognition closely depends on precision of iris segmentation. Problem in segmentation is having the same central point of iris as well as iris. Eyelids and eyelashes raise the problem in segmentation results in form of noise. Noise needs to be eliminated. For this proposed circular Hough transform to deduce the radius and finding center coordinates of pupil and iris region. In edge mapping, horizontal used for eyelids vertical used for finding the circular boundary of iris. Daugman Integro differential operator localizes the circular region of both and arcs defines the upper and lower eyelids. This operator is applied iteratively for precise localization. Eyelids are localized in similar with path of "contour integration" changed from circular to an arc. Active contour model has various vertices for localizing pupil changed its position with internal and external forces [2].

Cong, Zhang used 1D Gabor filter for separable and variance of intensity for multiple eyelashes for noise detection [2].

Padma Polash, Maruf Monwar presented an iris recognition system in order to verify together the uniqueness of the person iris and also its performance as a biometric identification. A biometric system provides automatic identification of an individual based on a unique feature or characteristic possessed by the individual. Iris recognition is regarded as the most reliable and accurate biometric identification system available. The iris recognition system consists of an automatic segmentation system that is based on the Hough transform, and is able to focus the circular iris 
and pupil region, occluding eyelids and eyelashes, and reflections. [3]

Himanshu Shrivastava worked for enhancement and denoising. Normalised iris image is still has low contrast and many images have non uniform illumination due positioning of light sources. To obtain better distributed texture image, enhance the image by local histogram equalization and remove high frequency noises by filtering the image. Hamming distance algorithm is in procedure for matching of iris with the stored template. Set the threshold value, if resultant value is greater than threshold, it declines else accepts [4].

Sowmya., Sreedevi.S.L describe recognition through iris sequence. For recognition first of all selection of clar image is required to outperform the iris image with low EER [8]. Recognition is performed under the either of or fusion of: texture based, appearance based and feature based. Feature based uses local variation which are characterized appearance and disappearance of an important image structure: PCA [8] is superior in image construction for feature extraction. By selecting commulative variance [8], it controls errors during construction of image. monochrome CCD is used for taking image from optical system. More abundant features [8] can be visible under Infra Red lightning. Captured image is in RGB form are converted into grayscale image. lowest number of dark pixels could be diameter of inner circle is MIN of histogram [8]. Scientists use CANNY detection for forming boundary and Hough for forming the circle [8]. Normalisation is used for generation of iris code and comparison. Segmentation normalises iris region. It reduces iris distortion.

Navjot Kaur, Mamta Juneja use retinex algorithm [24] as it removes illumination effects as noise provides columned constant image. non linear spectral transformation is used for synthesizing [24] the enhanced contrast of iris image. Kaur and Juneja use Fuzzy C mean clusters for making different clusters. Clusters are based on membership functions. One cluster is formed for high intensity value and other cluster is used for low intensity value. One other cluster is also used for in-between. For better edge detection and good segmentation, CANNY edge detector is used. to remove noise, morphological treatment is applied. Obtaining fine edges, circular hough transform [24] is applied. The process of recognition goes under retinex algorithm then fuzzy c mean then applied clustering techniques. Morphological operations is performed after clustering. To get iris area, vertical edge CANNY detection [24] technique is used. Hough transform is applied to bound the iris image. after applying mentioned techniques, iris template is created and then it is stored in associated database. Comparisons will be on the basis of hamming distance. Kaur and Juneja use MATLAB 8.0, windows 7s alogh with core 2duo processor. They use UBIRIS V2 as database.

Jaemin Kim described a new iris recognition algorithm, which uses a low level of details. Combining statistical classification and elastic boundary fitting, the iris is first localized. Then, the localized iris image is down-sampled by a factor of $\mathrm{m}$, and filtered by a modified Laplacian kernel. Since the output of the Laplacian operator is sensitive to a small shift of the full-resolution iris image, the outputs of the Laplacian operator are computed for all space-shifts. [25]

Ms. Rasika P. Rahane, Prof. Deepak Gupta studied for automatic identification with the help of biometric machines [26]. They focus on approaches of 1-D DWT (Discrete Wavelet Transform) filter execution, matching and recognition and circular normalization for recognition [26].

Tossy Thomas, Anu George, Dr.K P Indira Devi use RANSAC (Random Sample Consensus) to locate iris boundaries. RANSAC is more accurate than Hough transform. RANSAC is able to fit ellipse around non circular boundaries of iris. Duo scientists use Daugman's rubber sheet model and for template matching, they use PSR(Peak Side Lobe Ratio) is the similarity measure used for matching templates. [27]

\section{TECHNIQUES}

Three broad classification for iris recognition are: (1) Texture based [21] (2) Phase based [12] appearance based (3) Feature based extraction [21] (4) Intensity Variation Based Iris Recognition [22] (5) Cross Correlation Method For Feature Extraction Based Iris Recognition

\section{Texture Based Iris Recognition:}

Through the fusion of Haar Wavelet and Circular Mellin operator, the scientists obtained the texture based iris recognition [21]. To remove noises like holes, spots, the captured image is going under with preprocessing. Noises affect proper iris localization. Then processed image is localized through the inner and outer boundary detection of iris using maximum value of the spectrum image, the inner outer boundary of iris is to be calculated. scientists use Haar Wavelet and Circular Mellin operator. For obtaining rotation and scale invariant feature extraction, use Circular Mellin operator. The Haar Wavelet decomposition for the reduction of the size of feature vector, Haar Wavelet is used. then using Hamming distance, feature will compared. To calculate the overall result, fusion of duo techniques at decision level, Conjunction rule is used. used It results accurate more than 95\% Thus it can be considered as more robust[21]

\section{Phase Based Iris Recognition:}

Daugman integrodifferential operator [20] is used for inner and outer boundary of the iris. The estimation of center coordinates and radius is achieved through determination of maximum partial derivative of the contour integral of image. The path of contour integration [20] is altered from circular to arcuate for localizing eyelid boundaries.

\section{Feature Based Iris Recognition:}

Periocular region is used for the extraction of various appearance features. Some of Its perspectives are as:

(1) In the form of an independent biometric modality.

(2) To aid Iris recognition in non ideal situation $\{$ as a tool $\}$. 
Here computation of appearance features for each patch. In appearance based extraction, periocular image is splitting into spatial silent patches. To describe the entire image, the local features are combined. Various distance matrices are used for the computing the distance between the corresponding feature representation. This computation will match the images [21].

\section{Intensity Variation Based Iris Recognition:}

Hough Transform:

To determine parameters of geometric objects like lines and circles, a standard computer vision algorithm is used known as Hough transform [22]. To conclude the radius and center coordinates of pupil and iris region, circular Hough transform is used. Intensity variation [22] based iris recognition, automatic segmentation approach is used. By calculating the first derivatives of intensity value of image, an edge map is generated. Then the result will proceed through applying threshold. Edge points define better Hough space. Center coordinates and radius will correspond to a maximum point in the Hough space. Another type of named parabolic Hough transform [22] is used to detect eyelids. However Hough transform also has some of problems due to brute force attack [22]. So real time application may encounter the problem with recognition. For edge detection, Hough transform requires threshold. If critical edge points have to be remove then it would fail to detect circles.

\section{Cross Correlation Method For Feature extraction Based Iris Recognition:}

Template pixel values and source pixel values should be correlated [27]. Cross correlation is used for similarity findings between the template and test image.

\section{CHALLENGES AND OPEN ISSUES IN IRIS RECOGNITION}

We categorize issues of iris recognition systems in two broad classes namely those related to iris template security [16] and those associated with iris recognition performance. Details are as follows.

\section{A. Iris Template Security}

As biometric is an integral part of human body, loss of one's biometric corresponds to loss of his/her identity. Therefore, security of biometric templates is one of the most important concerns in any biometric authentication system. In literature, we found four types of biometric systems which are described below along with related issues and challenges.

\section{1) Traditional Biometric Systems}

These are the conventional systems which store users' templates in clear form to verify the identity. A template is generated at enrolment time, stored in the database without encryption/hashing and compared with the corresponding verification template at the verification time. As the template is used and stored in plaintext, a compromise of database has severe security and privacy implications. There are scenarios where users use the same biometrics for (c) 2015-19, IJARCS All Rights Reserved multiple applications or different organizations share data among themselves for their users. In such scenarios, crossmatching becomes feasible for tracking individual users [2], [3].

\section{2) Biometric Key Release}

These are the systems where biometrics along with cryptographic keys is used for authentication and communication [2]. The effort lies in using biometric templates effectively to release cryptographic keys in a secure manner. Modern cryptographic keys are uniformly random and large in size; therefore it is not feasible for users to memorize them. In biometric key release systems, cryptographic keys are stored at some location and are released using biometric information of the user. When user inputs his/her biometric, cryptographic key is released for use in any security protocol. This way, the key would be released only to the authorized users.

\section{3) Cancelable Biometrics}

For template security, These kind of systems apply some transformation on the biometric template. In these type of systems, the template is not stored directly in the system. Instead of that a function is applied on the template and the output of that function (transformed template) is stored in the database. To stopping the translation of original template into a compromised transformed template, transformation function must be non-invertible [16]. Strong reason behind using the cancelable biometric is that its original template is still remain secure even after transformed template has been compromised [16].

\section{4) Biometric Key Generation}

Except the biometric key generation system, biometric template and cryptographic key is stored in cleartext [16]. In biometric key generation systems, alliance of biometric template and cryptographic key is used to store to obtaining protection against leakage because bound value is not feasible to get the information [16].

\section{B. Iris Recognition Performance:}

The result of iris recognition can be compromised due to some of the following reasons:

\section{1) Dilation}

Dilation varies the size of pupil. Infirm pupil affects iris recognition performance. Drugs, light illumination, sunglasses play major role in pupil dilation. The factor of pupil dilation should be considered as a important point to secure from intrusion [16].

\section{2) Lenses}

Over 125 million people use contact lenses in our world. So iris recognition system should be accommodate with contact lenses users. Resultants are obtained in negative manner due to lenses. Lenses can affect the recognition by four ways: lenses have no effect even after their visibility, next results as light or dark outline around the sclera and iris. Another 
issue results as written logo or some symbol or text on the lenses itself and the last one comes with hard lenses [16].

\section{3) Time Variability}

Gonzalez' s study contradicts with the fact of iris stability through the life time [17]. However FAR is still remained unaffected while the FRR is increased. However obtaining more confidence on iris recognition, more research with more number of datasets is required to meet exact issue due to varying the time between the enrolling and verification.

\section{4) Cataract Surgery}

Dhir [18] experiments with 15 subjects and studied the differences between pre and post surgery in recognition. To explore the more accurate recognition due to surgery, more experiments with large number of datasets is required.

\section{5) System Portability}

FRR is higher when one sensor is used for enrolling and another one is used on the time of verification [19]. Researchers did their experiment with limited dataset and only IrisBEE software [19]. So more research with large number of datasets is required.

\section{DISCUSSION}

In this discussion part various Author had studied the iris recognition using bit plane slicing and normalization techniques, automated systems, iris recognition system in order to verify together the uniqueness of the person iris, Combining statistical classification and elastic boundary fitting fake iris detection method based on wavelet packet transform and accurate gait recognition from video is a complex presses involving heterogeneous features. To meet current security requirement, one of the biometric authentication method, i.e. iris recognition systems have found to be more accurate, compact, and efficient with satisfactory results because of rich texture of iris region and great variation of pattern among individuals.

Here, we had discussed various types of iris recognitions that can be launched against an iris recognition system. We have also discussed various challenges and importance of iris template protection techniques that can be used for a secure iris recognition system.

\section{REFERENCES}

[1] Srinivasa Kumar Devireddy, G.Ramaswamy, “A novel approach for an accurate human recognition us identification through iris recognition using bit plane slicing and normalization" Journal of Theoretical and Applied Information Technology 2005 - 2009 JATIT. All rights reserved.

[2] Matsoso Samuel Monaheng1, Padmaja Kuruba International Journal of Innovative Research in Science, "Iris recognition using circular hough transform", Engineering and Technology, Department Electronics and Communication, SET, Jain University, Bangalore, India1, Department Electronics and Communication, Global Academy of Technology, Bangalore, India2 (ISO 3297: 2007 Certified Organization), Vol. 2, Issue 8, August 2013, Copyright to IJIRSET www.ijirset.com 3546.
[3] Padma Polash, Maruf Monwar "Human iris recognition for biometric identification" Computer and information technology, 2007.iccit 2007. 10th international conference on10.1109/ICCITECHN.2007.4579354.

[4] Himanshu Srivastava, "Personal identification using iris recognition system, a review", International Journal of Engineering Research and Applications (IJERA) ISSN: 2248-9622.

[5] The University of Saskatchewan Department of Computer Science Technical Report \#2011-04.

[6] Marina Gavrilova, "Iris/retina biometrics. Lecture note on CPSC”, 601.20: Biometric Technologies, Department of Computer Science, University of Calgary, Canada. http://pages.cpsc.ucalgary.ca/ marina/601/.

[7] Nicholas M. Orlans John D. Woodward and PeterT.Higgins.Biometrics. McGraw-Hill/Osborne, New York, US, 2003.

[8] Sowmya., Sreedevi.S.L, "Iris recognition system for biometric identification”, EEE, Aarupadai Veedu Institute of Technology, VMUPaiyanoor,Chennai,India1sowmyakarthi@rediffmail.c om EEE, Aarupadai Veedu Institute of Technology, VMU Paiyanoor, Chennai,India 2second.author@second.com

[9] A.Bertillon, "la couleur de l'iris", Revue scientifique, France, 1885.

[10] Mayank Vatsa, Richa Singh, "Improving iris recognition performance using segmentation, quality enhancement, match score fusion, and indexing", ieee transactions on systems, man, and cybernetics-part b: cybernetics 1 student member, ieee, , student member, ieee, and afzel noore, member, ieee.

[11] Shejin Thavalengal, Petronel Bigioi, "Iris authentication in handheld devices -considerations for constraint-free acquisition”, Student Member, IEEE, , Senior Member, IEEE and Peter Corcoran, Fellow, IEEE.

[12] Surbhi Garg, Harmeet Kaur, "Survey paper on phase based iris recognition” Volume 4, Issue 4, April 2014 ISSN: 2277 128X International Journal of Advanced Research in Computer Science and Software Engineering.

[13] Kriti Sharma, Himanshu Monga, "Efficient biometric iris recognition using hough transform with secret key ", Volume 4, Issue 7, July 2014 ISSN: 2277 128X International Journal of Advanced Research in Computer Science and Software Engineering Research Paper.

[14] Gayatri Anand, Sachin Gupta, "A proficient graphical user interface based biometric iris recognition system", Volume 4, Issue 7, July 2014 ISSN: 2277128X International Journal of Advanced Research in Computer Science and Software Engineering.

[15] S V Sheela, Vijaya Malnad "201019 Iris recognition methods - survey", International Journal of Computer Applications (0975 - 8887)Volume 3 - No.5.

[16] Sajida Kalsoom, Sheikh Ziauddin "Iris recognition: existing methods and open issues", Department of Computer Science COMSATS Institute of Information Technology Islamabad, Pakistan Email: sajida.kalsoom@comsats.edu.pk Department of Computer Science COMSATS Institute of Information TechnologyI slamabad, Pakistan Email:s heikh.ziauddin@comsats.edu.pk

[17] P. Tome-Gonzalez, F. Alonso-Fernandez, and J. OrtegaGarcia, “Time variability in iris recognition”, IEEE International Conference on Biometrics: Theory, Applications and Systems, (BTAS), pages 1-6. IEEE, 2008.

[18] L. Dhir, N. Habib, D. Monro and S. Rakshit, "Effect of cataract surgery and pupil dilation on iris pattern recognition for personal authentication”, Eye, 24(6):10061010, 2009.

[19] K. Bowyer, S. Baker, A. Hentz, K. Hollingsworth, T. Peters, and P. Flynn., "Factors that degrade the match 
distribution in iris biometrics”, Identity in the Information Society, 2(3):327-343, 2009.

[20] Mehrotra, Hunny; Gupta, Phalguni; Kaushi, Anil K

[21] "Texture Based Iris Recognition System", Independent Component Analyses, Wavelets, Unsupervised NanoBiomimetic Sensors, and Neural Networks VI. Edited by Szu, Harold H.; Agee, F. Jack. Proceedings of the SPIE, Volume 6979, article id. 697903, 9 pp. (2008). (SPIE Homepage).

[22] Woodard, D.L., Pundlik, S.J., Miller, P.E.., “Appearancebased periocular features in the context of face and nonideal iris recognition”, Springer-VerlagPrint ISSN18631703Online ISSN1863-1711.

[23] Upasana Tiwari1, Deepali Kelkar2, Abhishek Tiwari3, "Study of different iris recognition methods", issn 22496343,international journal of computer technology and electronic engineering(ijctee) Volume 2, Issue 1.

[24] International Journal of Emerging Trends \& Technology in Computer Science (IJETTCS) -Special Issue ISSN 2278-6856, National Conference on Architecture, Software systems and Green computing-2013(NCASG2013) ISBN NO: 978-93-80609-14-0.

[25] Navjot Kaur, Mamta Juneja, "A novel approach for iris recognition in unconstrained environment”, UIET/CSE
Department, PU Chandigarh, India, Email: navjotkaur2611@gmail.comUIET/CSE Department, PU Chandigarh, India, Email: mamtajuneja@pu.ac.in.

[26] Jaemin Kim, "Iris recognition using a low level of details", School of Electronics and Electrical Engineering, Hongik University,72-1 Sangsu-dong, Mapo-gu, Seoul 121-791, Korea, 2006.

[27] Ms. Rasika P. Rahane, Prof. Deepak Gupta, "The literature survey on latest iris recognition technique”, IJARCCE ISSN (Online) 2278-1021ISSN (Print) 2319 5940, International Journal of Advanced Research in Computer and Communication Engineering,ISO 3297:2007 Certified,Vol. 6, Issue 1, January 2017, Copyright to IJARCCE DOI 10.17148/IJARCCE.2017.6129 149

[28] Tossy Thomas,Anu George,Dr.K P Indira Devi, "Effective iris recognition system ”, ScienceDirect2212-0173 (C) 2016 Published by Elsevier Ltd. This is an open access article under the CC BY-NC-ND license (http://creativecommons.org/licenses/by-nc-nd/4.0/).Peerreview under responsibility of the organizing committee of RAEREST 2016 doi: 10.1016/j.protcy.2016.08.133 Global Colloquium in Recent Advancement and Effectual Researches in Engineering, Science and Technology (RAEREST 2016). 